\title{
Comparison of real-time visualization of volumetric OCT data sets by CPU-slicing and GPU-ray casting methods
}

\author{
Alfred R. Fuller ${ }^{a}$, Robert J. Zawadzki ${ }^{b^{*}}$, Bernd Hamann ${ }^{a}$ and John S. Werner ${ }^{b}$ \\ ${ }^{a}$ Visualization and Computer Graphics Research Group, Institute for Data Analysis and \\ Visualization (IDAV), Department of Computer Science, UC Davis, One Shields Avenue, \\ Davis, CA 95616, USA; \\ ${ }^{b}$ Vision Science and Advanced Retinal Imaging Laboratory (VSRI) and Department of \\ Ophthalmology \& Vision Science, UC Davis, 4860 Y Street, Suite 2400, Sacramento, CA \\ 95817, USA;
}

\begin{abstract}
We describe and compare two volume visualization methods for Optical Coherence Tomography (OCT) retinal data sets. One of these methods is CPU-slicing, which is previously reported and used in our visualization engine. The other is GPU-ray casting. Several metrics including image quality, performance, hardware limitations and perception are used to grade the abilities of each method. We also discuss how to combine these methods to make a scalable volume visualization system that supports advanced lighting and dynamic volumetric shadowing techniques on a broad range of hardware. The feasibility of each visualization method for clinical application as well as potential further improvements are discussed.
\end{abstract}

Keywords: Optical coherence tomography, imaging system, medical optics instrumentation, ophthalmology, volume visualization

\section{INTRODUCTION}

Recent progress in Fourier domain-Optical Coherence Tomography (Fd-OCT) $)^{1-5}$ has allowed for successful implementation for ophthalmic clinical applications. ${ }^{6-8}$ The fast acquisition speeds of Fd-OCT have led to the volumetric imaging of in vivo retinal structures resulting in large volumetric data sets. Thus there is growing demand for volume visualization and manipulation software. Following this direction over last three years our group has developed custom volume visualization software. ${ }^{9}$ This software has proven to be a powerful tool for conveying the information acquired using Optical Coherence Tomography (OCT) through computer visualization.

The goal of computer visualization is to convey meaningful information about a data set through a visual representation. This is done by simulating stimuli on a computer screen that the human brain is accustomed to interpreting. However, computer visualization is limited by the resources of the computer generating the stimuli. This means that it can be costly in terms of computer processing power and resources to produce physically accurate stimuli akin to the natural world we experience and interpret every day. By dividing the perceptual experience into specific visual cues and targeting the specific perceptions associated with these cues, computer visualization has been able to find a middle ground between limited computer resources and the desired conveyance of information. Naturally, as computers advance, this middle ground is continually being pushed forward to produce more meaningful visualizations.

Volume visualization of OCT retinal data sets strives to convey composition, shape, structure, and relative depth and size of various retinal structures. The basic method behind volume visualization uses a transfer function to convert the data values to visual stimuli. Visual stimuli are generated via color and opacity values which are projected onto a computer screen to convey the basic composition of the data set. Additional visual cues are added to further enhance the user's perception of the data set.

\footnotetext{
*rjzawadzki@ucdavis.edu; phone 1916 734-5839; fax 1916 734-4543; http://vsri.ucdavis.edu/
} 
There are several significant visual cues that we consider for this particular application of volume visualization. A perspective projection of the visual stimuli enhances the user's perception of both size and depth. ${ }^{10}$ Smoothly animated user-guided manipulation of the visualization allows the user to explore the composition of the data set and enhances the perceived relative depth and size of different structures within the data set. Advanced lighting models enhance the perceived shape of these structures while shadows further enhance their relative depth and size. Smooth animation and manipulation has been achieved with hardware-accelerated volumetric rendering and is widely used. Proper perspective is partially achieved with the current commonly used volume visualization techniques while advanced lighting is rarely implemented and shadows are almost never used due to the large cost associated with their computation.

We examine two volume visualization techniques that support these types of visual cues, CPU(central processing unit)-slicing and GPU(graphics processing unit)-ray casting. CPU-based methods are executed on the main computer's processing unit which typically supports between one and four simultaneous operations. GPU-based methods are executed on the graphic card's processing unit which can support anywhere from 16 to 512 (GeForce 9800 GX2 SLI) simultaneous operations. CPU-Slicing was originally introduced by Cullip and Neumann ${ }^{11}$ and Cabral et al. ${ }^{12}$ and has since been improved ${ }^{13-15}$ while the first single pass GPU-ray casting framework was introduced by Stegmaier et al. ${ }^{16}$

As is usually the case in computer visualization, the use of these techniques is primarily limited by the available computer resources. Lighting and shadows have generally been excluded from volume visualizations so that smooth animation and manipulation can be archived given these limitations. However, we show that these particular visual cues can reasonably be implemented and combined into a single visualization on modern graphics hardware. Additionally, as both rendering methods have many implications with regard to their viability in a clinical setting, we describe a robust solution that utilizes both rendering methods to maximize the performance and scalability of our overall system.

Section 2.1 describes the basic ideas behind CPU-slicing while Section 2.2 demonstrates how we can use GPU-ray casting to improve upon CPU-slicing when modern graphics hardware is available. However, GPUray casting also has another application. In Section 3.1 we describe a method that uses GPU-ray casting to implement dynamic volumetric shadows. This implementation can be used with normal GPU-ray casting as well as CPU-slicing. In Section 4.2 we discuss how these methods can be combined to scale to nearly any hardware configuration. Section 4.3 extends upon this scalability to accommodate the needs of specific user activities.

\section{RENDERING METHODS}

\subsection{CPU-slicing}

Both CPU-slicing and GPU-ray casting implement evenly spaced sampling volume reconstruction. ${ }^{17}$ However, the method by which the positions of these samples are calculated varies. CPU-slicing generates two-dimensional slices of the volume perpendicular to the central view vector on the CPU and reconstructs the volume using alpha blending on the GPU. Figure 1 shows a simplified two-dimensional representation of CPU-slicing. These slices are known as "proxy geometry," as their only function is to trigger a program known as a "shader" to run on the GPU at the given position. The shader samples the volume and maps the data value to a color and opacity (alpha value) through a transfer function like the one seen in Figure 2. As the user changes the orientation of the volume, the CPU must re-slice and re-render the entire volume. Figure 3 shows the same simplified two-dimensional representation as Figure 1 except with the volume rotated.

As the sampling of the volume occurs on the graphics card, the GPU needs access to the volumetric data. However, graphics cards are limited by the amount of on-board memory available and OCT data sets tend to exceed this limit. To overcome this constraint, the volumetric data set is divided into data blocks of fixed size, which are rendered individually. ${ }^{18}$ Figure 3 shows a two-dimensional example of these data blocks. Each region in Figure 3 must be sliced and rendered separately, greatly increasing the amount of work the CPU must perform before the volume can be reconstructed on the GPU. Figure 4 show a small portion of the slices generated for a real OCT data set.

The basic equation that governs volumetric rendering is $A(t, x)=1-e^{\int_{0}^{t} s\left(x+t^{\prime}\right) d t^{\prime}}$ where $x$ is a position in space, $t$ is the spacing between adjacent samples and $A$ is the opacity or alpha value accumulated from $x$ to 


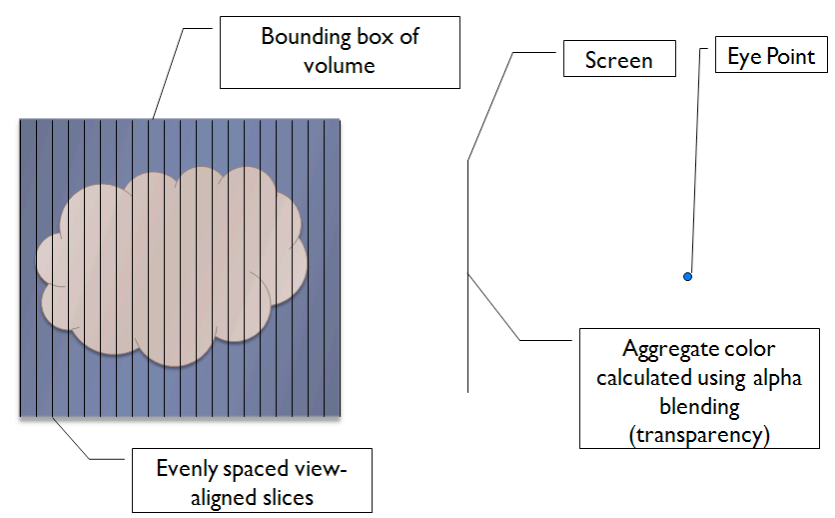

Figure 1. A two-dimensional representation of the CPU-slicing algorithm. The CPU generates view-aligned polygons (or slices) at evenly spaced intervals throughout the bounding box of the volume. The graphics card projects these slices onto the screen. For each screen pixel that is covered by a projected slice, the GPU runs a "shader" that samples the volumetric data and returns an associated color and opacity. Alpha blending is used to aggregate theses colors and opacities for each pixel.

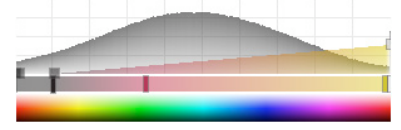

Figure 2. An example of a transfer function from our application. A one-dimensional transfer function maps a data value to a color and opacity for use in volumetric visualizations. The top region controls the opacity while the middle region controls the color. The bottom region is used by the user to specify a color. A histogram of the data set is provided in the background of the top region to guide the user in the selection of a meaningful transfer function.

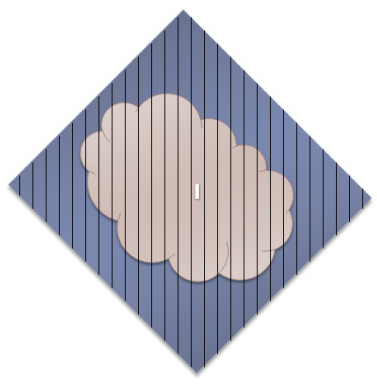

(a)

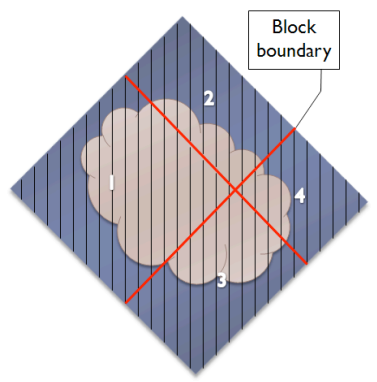

(b)

Figure 3. (a) is the same volume as shown in Figure 1 except rotated and re-sliced. A volume must be re-sliced by the CPU for every change in orientation. (b) is this same rotated volume, but divided into separately rendered blocks. In this case, the volume must be re-sliced four times by the CPU for every change in orientation. The OCT data sets presented in this paper contain about 160 of these blocks that need to be re-sliced for every change in orientation. 


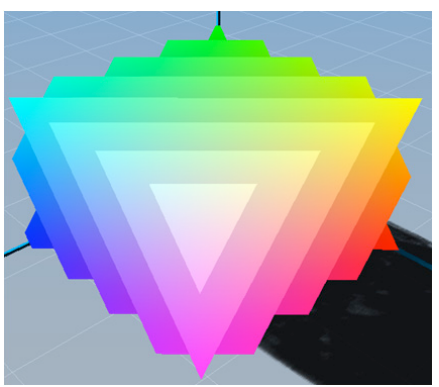

(a)

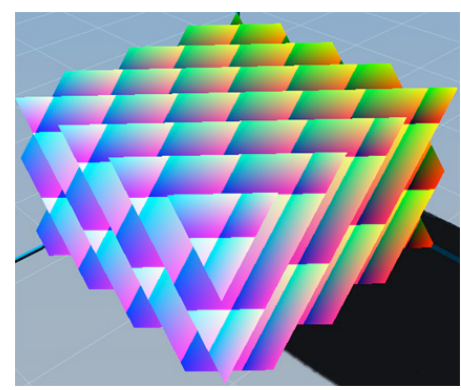

(b)

Figure 4. A small subset of the samples used in CPU-slicing to render a real OCT volumetric data set. Each color denotes the location in the volumetric data set that is sampled through the relationship $(x, y, z)=($ red,green, blue). The samples in (b) are from a volume divided into separately rendered data blocks. The coloring of the blocked volume shows that each block is rendered with its own local data space coordinates.

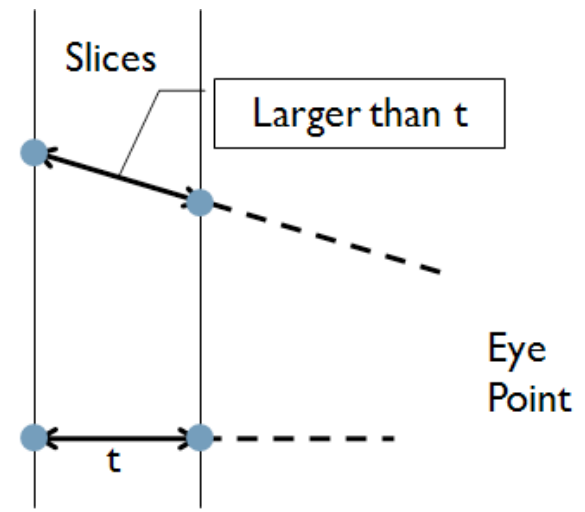

Figure 5. Two pixels sampling two slices each from the CPU-slicing algorithm. The farther from the center of the screen a pixel is, the less accurate our assumed constant $t$ value becomes. Each dot represents a sample of the volumetric data set and each line represents the path of samples for a single pixel.

$x+t .^{19}$ In order to efficiently use this equation on the GPU we apply two approximations. First we set $s(x)$ to be a constant between $x$ to $x+t$. This eliminates the integral and $A(t, x)=1-e^{s(x) * t}$. Then we set $t$ to be a constant which causes $A$ to only be a function of $x$. With these simplifications we can accumulate color contributions from back to front with the recursive equation $C_{i}=C(x) * A(x)+(1-A(x)) * C_{i-1}$ where $C_{i}$ is the color after accumulating the $i$ th sample. To perform this operation we only need to convert a point in space, $x$, into a color and opacity. This is done in a GPU shader by sampling the volume at point $x$ and using a user-defined transfer function such as the one depicted in Figure 2 to convert the sampled value to a color and opacity. Unfortunately when we consider the samples used by CPU-slicing to reconstruct the volume we find that a perspective projection of the slices does not maintain a constant $t$-value as exemplified in Figure 5 . As a result of this inaccuracy the volumetric rendering can appear faded on the periphery of the visualization. This fading scales with distance from the center of the image and sample spacing. One of the advantages of GPU-ray casting is that it does not suffer from this problem.

\subsection{GPU-ray casting}

As a replacement for CPU-slicing, GPU-ray casting uses the GPU to calculate a ray from the eye point to the volume for each pixel and samples the volume along these rays. This is done by generating proxy geometry on the front-facing boundary of the volume, which causes a GPU shader to execute along this boundary. While the shader used in CPU-slicing is only responsible for sampling a single point in the data, the shader used in GPU-ray casting both samples all points needed by a given pixel and accumulates the values. These samples are performed on a fixed interval of length $t$ as seen in Figure 6. Figure 7 shows a small subset of the samples used in GPU-ray casting for a real OCT data set both with and without blocking. These samples occur on the surface of concentric spheres centered at the eye point with each adjacent sphere being exactly $t$ apart. This is 


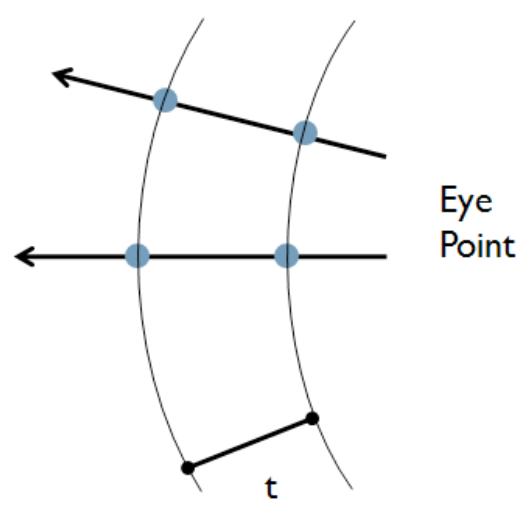

Figure 6. Two rays from GPU-ray casting. These rays originate at the eye point and travel through different pixels on the screen and into the volume. Each ray samples the volume on the same fixed interval, $t$. Each arrow represents a ray and each dot represents a sample of the volume.

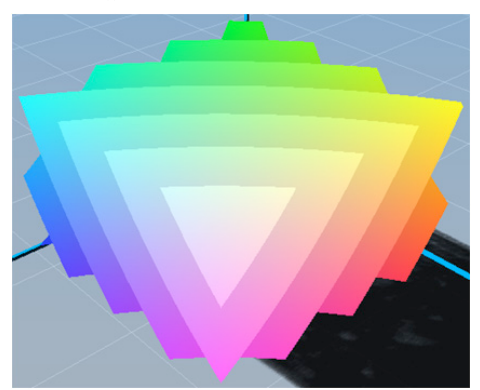

(a)

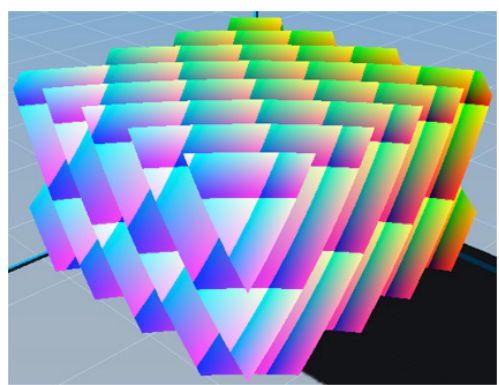

(b)

Figure 7. A small subset of the samples used in GPU-ray casting to render a real OCT volumetric data set. Each color denotes the location in the volumetric data set that is sampled through the relationship $(x, y, z)=($ red,green,blue $)$. The samples in (b) are from a volume divided into separately rendered data blocks. Note that these samples occur on the surface of concentric spheres instead of on parallel planes as seen in Figure 4.

distinctly different from CPU-slicing, where samples occur on parallel planes that are spaced $t$ apart as seen in Figure 4. By maintaining a constant $t$-value, GPU-ray casting provides a better approximation to the volume rendering equation when using a perspective projection. Additionally, since no slicing is required, the $\mathrm{CPU}$ is only tasked with specifying the boundary of the volume. However, since the shader used in GPU-ray casting is far more complex than the one used in CPU-slicing, it requires a more capable graphics card.

GPU-ray casting allows for exact early ray termination. Since the shader used in GPU-ray casting is responsible for all the samples along a single ray, it can detect when further samples will produce a negligible contribution to the final color accumulated along a ray. It can stop sampling the volume early and still return an accurate result. Figure 8 shows the length along each ray that was sampled for the given volume.

\section{VISUAL ENHANCEMENTS}

\subsection{Dynamic Volumetric Shadows}

The visibility function, $v(x)$ is used to incorporate shadows into a lighting model. The visibility function, $v(x)$, returns one to indicate full light and zero to indicate full darkness. Figure 9 shows this function on a real OCT data set. Our application calculates this function directly for each sample by using the GPU to cast "shadow feeler" rays towards the light source. The opacity of the volume is sampled along these rays and accumulated to find the percentage of light that is not occluded. Figure 10 depicts this process. This method has traditionally been considered too slow to preserve interactivity of the application, but our application is able to overcome this computational barrier by utilizing the GPU to cast these rays and implementing several methods to accelerate the computation of these rays. 


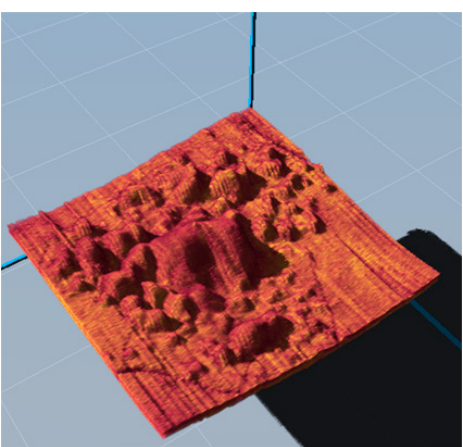

(a)

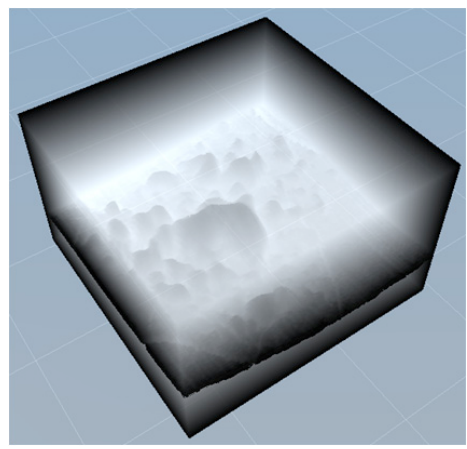

(b)

Figure 8. (a) is a normal GPU-ray casting rendering of an OCT data set while (b) shows the lengths of each ray used to render the image in (a). In (b) shorter rays are dark while longer rays are light. Rays terminate at the front opaque surface of the volume through the use of early ray termination. This approach can greatly reduce the number of samples needed to reconstruct the volume.

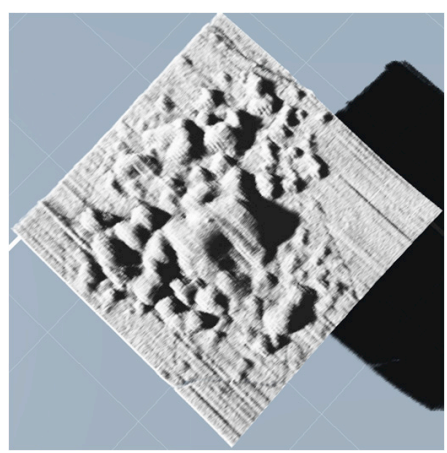

(a)

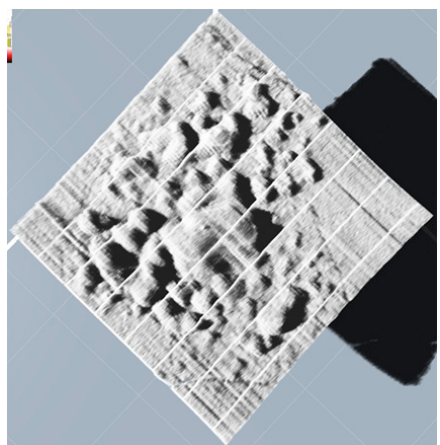

(b)

Figure 9. Rendering of the visibility function for both an unblocked (a) and a blocked (b) volume. The visibility function, $v(x)$ is one in full light and zero in full darkness. The visibility function uses GPU-based "shadow feeler" rays to determine how visible the light source is at a given point. These rays are restricted to the data in graphics memory, which explains why (b) shows discontinuities when occluding structures are in external data blocks.

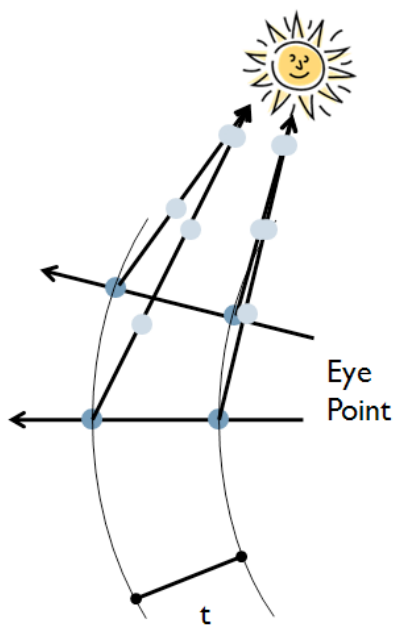

Figure 10. Two rays from GPU-ray casting that also cast "shadow feeler" rays. These rays sample the volume to determine how much light is visible at the given sample point. Casting these rays greatly increases the time it takes to render the volume. However, this cost can be mitigated through the use of several optimizations of the ray casting algorithm. Each arrow represents a ray and each dot represents a sample of the data set. The "shadow feeler" rays can also be cast when using CPU-slicing on certain hardware. 


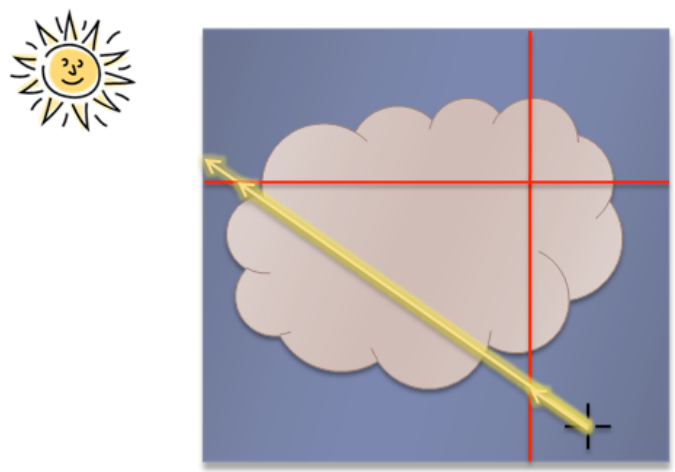

(a)

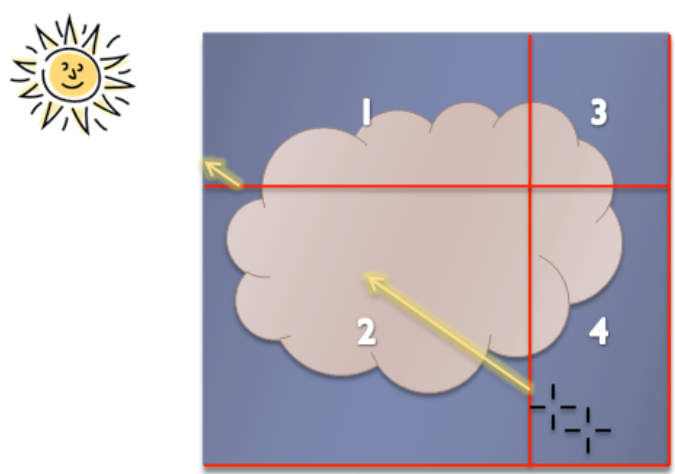

(b)

Figure 11. (a) shows the rays needed to calculate the visibility function at the given point when rendering separate data blocks. (b) shows the rays used when optimized shadow caching and early ray ternimation are applied for the same point. (b) also shows an additional point that requires no extra work to determine its visibility function. The ray in region 2 of (b) terminates early as its visibility function is zero before reaching the end of the data. Region 4 of (b) never needs to cast a shadow feeler ray as the value on the border between regions 2 and 4 already represents full darkness.

As with the normal GPU-ray casting, we can also use early ray-termination to speed up these shadow feeler rays. Additionally, the accuracy of the shadow feeler rays tends not to influence the visual fidelity of the resulting image as much as rays used to reconstruct the volume. With this in mind we can increase the sample spacing, $t$, of the shadow feeler rays to something much larger than the $t$ used to reconstruct the volume. Our application defaults to a shadow sample spacing five times that of the normal sample spacing. We have also added the ability to further increase this sample spacing dynamically on the GPU when sampling transparent regions of the volume.

This method fails when the data set is divided into blocks that are rendered separately. In this case, the shadow feeler ray cast at a given location only has access to a sub-region of the data. This causes problems when the location in question is shadowed by structures external to the available data. Figure 8 shows the resulting visibility function of a blocked data set. To solve this problem our application uses a shadow cache. This cache stores intermediate values of the visibility function on the boundaries of blocked regions. This cache is made available to the separate sub regions of the volume and restores the continuity of the visibility function. Each shadow feeler ray is now actually divided into several different rays which are cast separately and accumulated for each sample point as seen in Figure 11. This approach has the added benefit of greatly reducing the amount of redundant sampling occurring and drastically limits the length of shadow feeler rays that are cast during rendering. In the example presented in Figure 11(b), the points highlighted in region 4 never cast a shadow feeler ray because the shadow cache already indicates full darkness. Figure 12 compares various volumes with and with out shadows.

\subsection{Advanced Lighting}

Any per-sample lighting model can be used to add lighting to both CPU-slicing and GPU-ray casting. There are many different optical models ${ }^{19}$ that can be used. In our program, we have implemented one of the most popular lighting models, the Blinn-Phong model. ${ }^{20}$ This lighting model is ideal for volumes that contain well-defined surfaces, as it is meant to approximate a micro-faceted surface and needs a normal vector at each sample point. These normals can be extracted by the GPU on the fly or pre-calculated and uploaded along with the volume data. Since computing this normal on the fly introduces a large overhead and the normal values remain relatively constant, our application pre-calculates, smooths and down samples the normals. Figure 13 shows a rendering of a pre-computed normal map. The calculation of these normals can be done on the CPU or more efficiently on the GPU. Technically these normals change when the opacity portion of the transfer function changes. However since changes in opacity usually only represent changes in the magnitude of the gradient vector, in most cases these normal vectors can be calculated once for each data set. The result of pre-calculating the normals, uploading them to graphics memory and using the Blinn-Phong lighting model on the GPU can be seen in Figure 14(b). Figure 14(c) shows a volume rendered with both Blinn-Phong lighting and shadows. 


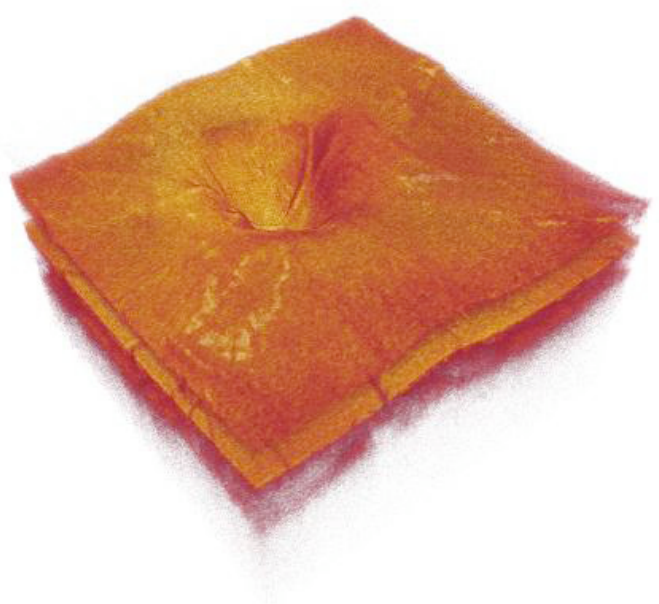

(a)

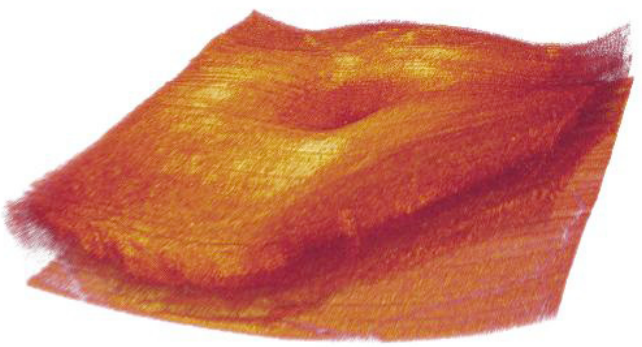

(c)

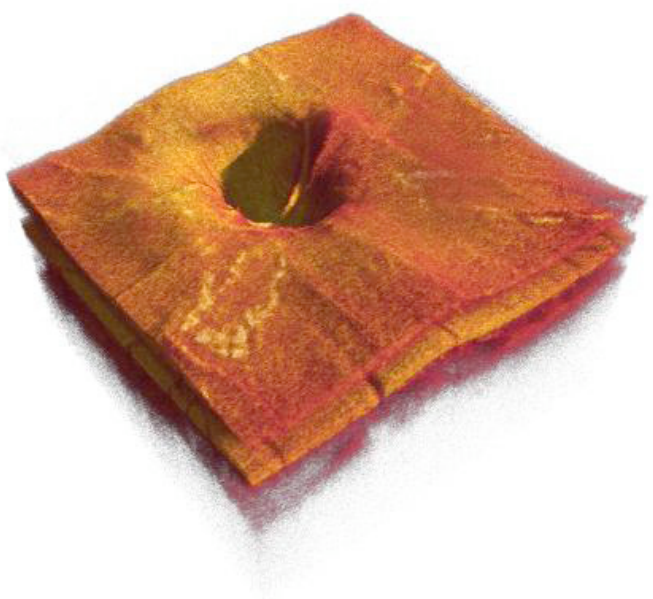

(b)

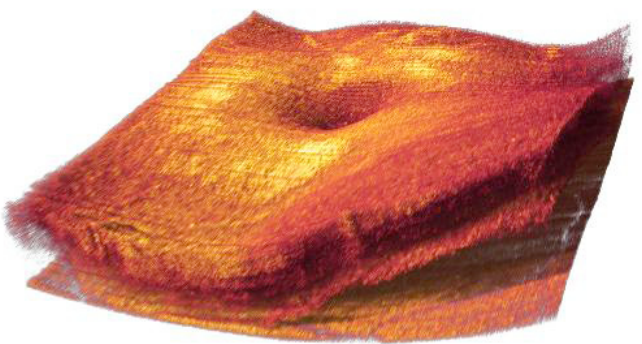

(d)

Figure 12. A comparison of various volumes with and with out shadows. (b) and (d) are using GPU-based "shadow feeler" rays to cast shadows. (a) and (b) show a healthy retina while (c) and (d) illistrate retinal detachment.

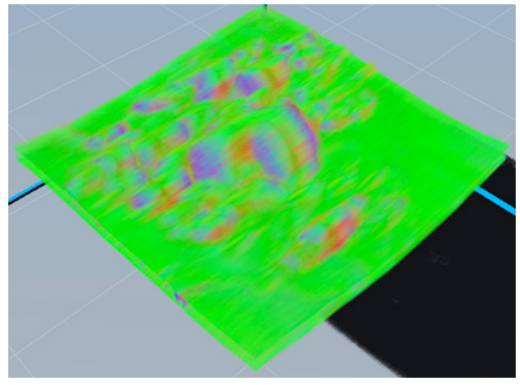

Figure 13. The normal map extracted from the volume seen in Figure 8(a). It has been smoothed and down-sampled to improve the visual quality and reduce its memory footprint. A normal map associates each voxel in the volumetric data set to a normal vector. In this image, the normal vector $(x, y, z)$ is represented by (red, green,blue). 


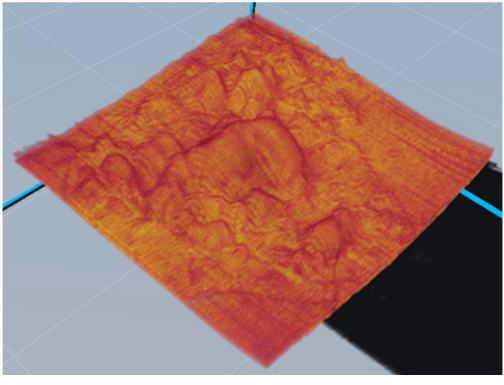

(a)

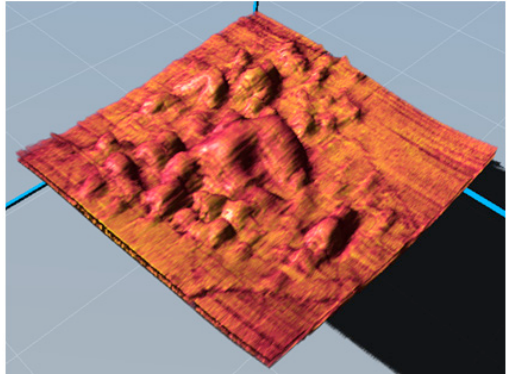

(b)

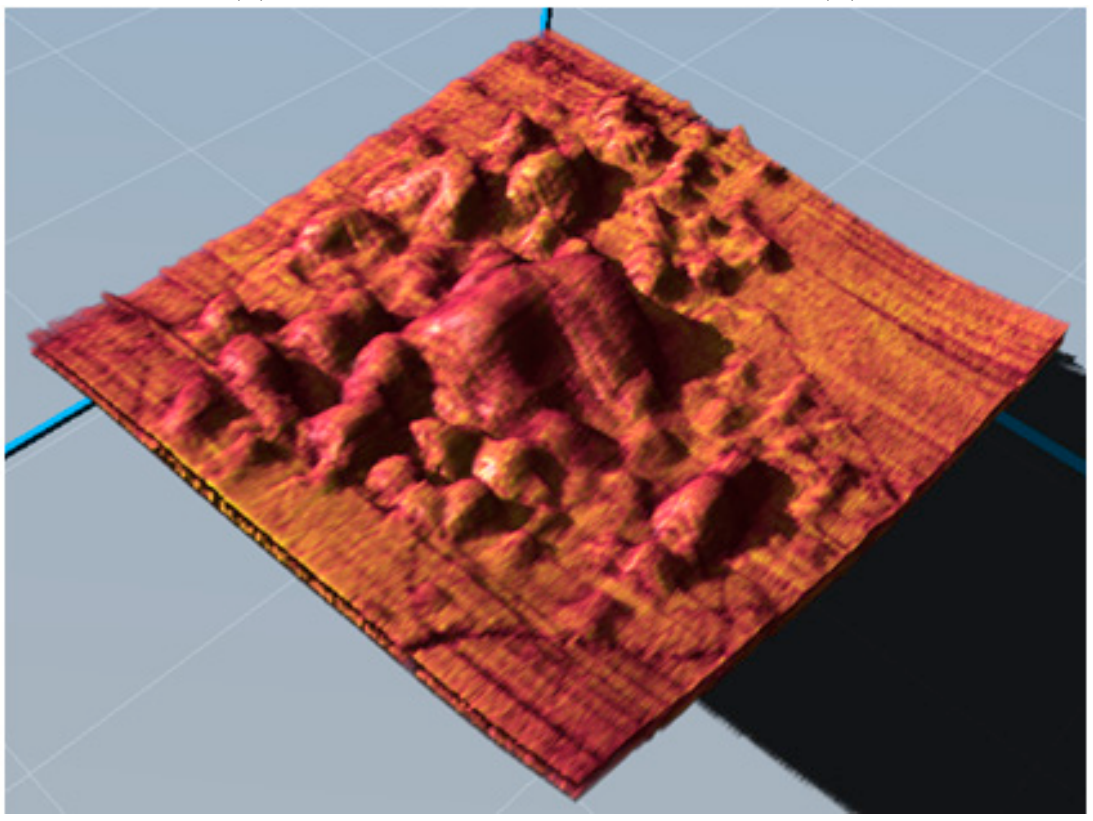

(c)

Figure 14. Rendering of the photoreceptor layer of a retina suffering from macular degeneration. (a) has no lighting or shadows and is representative of the common form of volumetric visualization. (b) uses Blinn-Phong lighting model. (c) uses both Blinn-Phong lighting and shadows. In this case the size, shape and relative position of the drusen is not easily perceived in (a) without rotating the volume. The visual cues added to (b) and (c) greatly increase the amount of information that is conveyed to the viewer in a single image. 


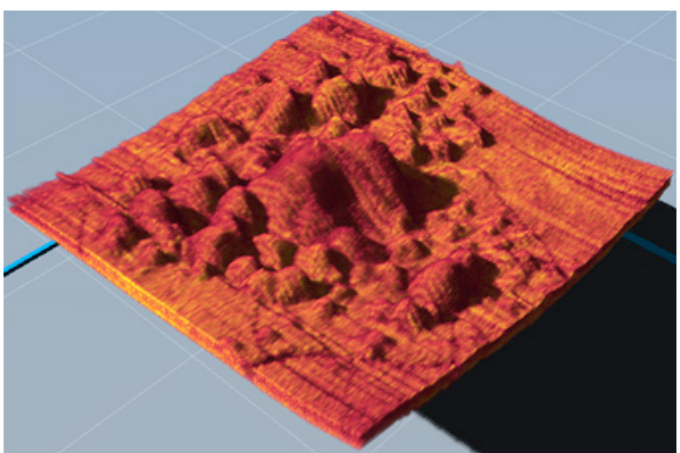

(a)

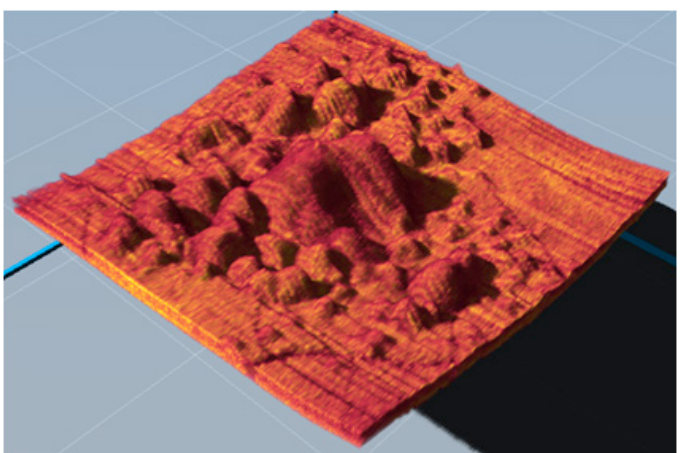

(b)

Figure 15. A comparison of CPU-slicing (a) and GPU-ray casting (b) when both use GPU-based "shadow feeler" rays to cast shadows. With small enough sample spacing, the visual difference is negligible but performance and hardware requirements of these methods differ.

\section{PERFORMANCE SCALING}

\subsection{Comparison}

Figure 15 shows the same volume rendered both with CPU-slicing and GPU-ray casting. These images are nearly identical in appearance but differer in their performance and hardware requirements. The GPU-ray casting renders faster while the CPU-slicing is supported by a wider range of graphics cards. On the volume in Figure 15, which occupies 600x500 pixels and is reconstucted from a 997x350x97 data set scaled to 997x700x970, our application performs CPU-slicing in 100ms (10fps) and GPU-ray casting in 50ms (20fps) with a simple spacing of one sample per voxel and full lighting and shadows. From experience 100ms (10fps) - 66ms (15fps) is adequate for interactive medical applications. On top of this there are many known algorithmic optimizations that we have yet to implement in our system such as global empty space skipping and global early ray termination ${ }^{15}$ that can improve both CPU-slicing and GPU-ray casting.

\subsection{Hardware Limitations}

Table 1 provides an example of common hardware limitations and the available rendering methods associated with each configuration. Since our system can use both CPU-slicing and GPU-ray casting individually or in combination (CPU-slicing with GPU-shadows feeler rays) we can utilize the full resources of any system. Our current framework supports fixed function graphic processors as well as the latest graphic cards, thus we can visualize volumetric data sets on relatively old laptops in addition to new high-end desktop computers.

\begin{tabular}{|l|l|l|l|}
\hline Pixel Shader & Min nVidia Generation & Year Released & Capabilities \\
\hline Fixed Function & $<3$ Series & $<2001$ & $\begin{array}{l}\text { CPU Slicing } \\
\text { Grayscale } \\
\text { Some TF }\end{array}$ \\
\hline PS 2 & 3 Series & 2001 & Full TF \\
\hline PS 2.a & 5 Series (FX) & 2003 & Lighting \\
\hline PS 4 & 7 Series & 2005 & GPU Ray Casting or Shadows \\
\hline PS 4+ & 8 Series & 2007 & GPU Ray Casting and Shadows \\
\hline
\end{tabular}

Table 1. This table lists the minimum pixel shader version and nVidia chip generation required to use different capabilities of our system. Most desktops operating today will support at least PS 2.a while PS 4+ is only supported on high-end computers bought after 2006.

\subsection{Useablility Scaling}

Using the latest and most costly visualization technology is not always the best approach. This is the case with some slower computers that support shadows, as rendering these shadows may increase the rendering time to sub-interactive levels. To address this issue, we have divided user-driven activities into six different categories and 
allow the user to customize the visualization for each activity as seen in Table 2. One such customization might be to disable shadows for fast animation while the user is manipulating the volume, and re-enabling shadows once the user has found an ideal viewing angle. For ease of use, each activity has a base activity from which the user only needs to specify changes in settings. This strategy also allows for a smooth transition between activities. For example, when rotating a volume, one might want to scale the sample spacing based on the speed of rotation in such a way that the image at rest is identical to the image moving at a speed of zero. This would increase animation speed while manipulating the volume with no discontinuity in the transition from a static orientation.

\begin{tabular}{|l|l|l|}
\hline Activity & Description & Default Base Activity \\
\hline Base & Default values & N/A \\
\hline Still & The volume and light source are stationary & Base \\
\hline Transfer Function Changing & The transfer function is being actively edited & Still \\
\hline Volume Moving & The volume is in motion & Still \\
\hline Light Moving & The light source is in motion & Still \\
\hline Screen Capture & A single frame is being extracted & Base \\
\hline Video Capture & Multiple frames of animation are being captured & Screen Capture \\
\hline
\end{tabular}

Table 2. A table of user-driven activities. Each activity provides its own set of modifications from its base activity. These settings can be customized to fit the preferences of the user, the limitations of the hardware, and the needs of the activity.

\section{FUTURE WORK}

We plan to implement additional optimizations such as global early ray termination and global empty space skipping for both CPU-slicing and GPU-ray casting so that we can see where the true performance limit of our method lies. We also would like to automate the configuration of our implementation so that the user can simply load a representative data set and have the application execute a series of performance tests to automatically find the best configuration for the user's specific hardware.

\section{ACKNOWLEDGMENTS}

We gratefully acknowledge the support made available to Alfred Fuller through the Lawrence Scholar Program of Lawrence Livermore National Laboratory. This research was also supported by the National Eye Institute (EY 014743).

\section{REFERENCES}

1. A. F. Fercher, C. K. Hitzenberger, W. Drexler, G. Kamp, and H. Sattmann, "In vivo optical coherence tomography," Am. J. Ophthalmol. 116, pp. 113-114, 1993.

2. M. Wojtkowski, R. Leitgeb, A. Kowalczyk, T. Bajraszewski, and A. F. Fercher, "In vivo human retinal imaging by fourier domain optical coherence tomography," Journal of Biomedical Optics 7, pp. 457-463, 2002 .

3. N. Nassif, B. Cense, B. Park, M. Pierce, S. Yun, B. Bouma, G. Tearney, T. Chen, and J. de Boer, "In vivo high-resolution video-rate spectral-domain optical coherence tomography of the human retina and optic nerve," Optics Express 12, pp. 367-376, 2004.

4. B. Cense, N. Nassif, T. Chen, M. Pierce, S.-H. Yun, B. H. Park, B. Bouma, G. Tearney, and J. de Boer, "Ultrahigh-resolution high-speed retinal imaging using spectral-domain optical coherence tomography," Optics Express 12, pp. 2435-2447, 2004.

5. M. Wojtkowski, V. Srinivasan, T. Ko, J. Fujimoto, A. Kowalczyk, and J. Duker, "Ultrahigh-resolution, high-speed, fourier domain optical coherence tomography and methods for dispersion compensation," Optics Express 12, pp. 2404-2422, 2004.

6. M. Wojtkowski, V. Srinivasan, J. Fujimoto, T. Ko, J. Schuman, A. Kowalczyk, and J. Duker, "Threedimensional retinal imaging with high-speed ultrahigh-resolution optical coherence tomography," Ophthalmology 112, pp. 1734-1746, 2005. 
7. U. Schmidt-Erfurth, R. A. Leitgeb, S. Michels, B. Povazay, S. Sacu, B. Hermann, C. Ahlers, H. Sattmann, C. Scholda, A. F. Fercher, and W. Drexler, "Three-dimensional ultrahigh-resolution optical coherence tomography of macular diseases," Invest. Ophthalmol. Vis. Sci. 46, pp. 3393-3402, 2005.

8. S. Alam, R. J. Zawadzki, S. S. Choi, C. Gerth, S. S. Park, L. Morse, and J. S. Werner, "Clinical application of rapid serial fourier-domain optical coherence tomography for macular imaging," Ophthalmology 113, pp. 1425-1431, 2006.

9. R. Zawadzki, A. Fuller, D. Wiley, B. Hamann, S. Choi, and J. Werner, "Adaptation of a support vector machine algorithm for segmentation and visualization of retinal structures in volumetric optical coherence tomography data sets," Journal of Biomedical Optics 12, pp. 041206 (1-8), 2007.

10. D. Vishwanath, A. Girschick, and M. Banks, "Why pictures look right when viewed from the wrong place," Nature Neuroscience 8, pp. 1401-1410, 2005.

11. T. J. Cullip and U. Neumann, "Accelerating volume reconstruction with $3 \mathrm{~d}$ texture hardware," tech. rep., Chapel Hill, NC, USA, 1994.

12. B. Cabral, N. Cam, and J. Foran, "Accelerated volume rendering and tomographic reconstruction using texture mapping hardware," in VVS '94: Proceedings of the 1994 symposium on Volume visualization, pp. 91-98, ACM Press, (New York, NY, USA), 1994.

13. R. Westermann and T. Ertl, "Efficiently using graphics hardware in volume rendering applications," in SIGGRAPH '98: Proceedings of the 25th annual conference on Computer graphics and interactive techniques, pp. 169-177, ACM Press, (New York, NY, USA), 1998.

14. C. Rezk-Salama, K. Engel, M. Bauer, G. Greiner, and T. Ertl, "Interactive volume on standard pc graphics hardware using multi-textures and multi-stage rasterization," in $H W W S$ '00: Proceedings of the ACM SIGGRAPH/EUROGRAPHICS workshop on Graphics hardware, pp. 109-118, ACM, (New York, NY, USA), 2000.

15. W. Li, K. Mueller, and A. Kaufman, "Empty space skipping and occlusion clipping for texture-based volume rendering," in VIS '03: Proceedings of the 14th IEEE Visualization 2003 (VIS'03), IEEE Computer Society, (Washington, DC, USA), 2003.

16. S. Stegmaier, M. Strengert, T. Klein, and T. Ertl, "A simple and flexible volume rendering framework for graphics-hardware-based raycasting," in Volume Graphics, pp. 187-195, 2005.

17. J. T. Kajiya and B. P. VonHerzen, "Ray tracing volume densities," SIGGRAPH Computer Graphics 18(3), pp. $165-174,1984$.

18. P. Bhaniramka and Y. Demange, "Opengl volumizer: a toolkit for high quality volume rendering of large data sets," in VVS '02: Proceedings of the 2002 IEEE symposium on Volume visualization and graphics, pp. 45-54, IEEE Press, (Piscataway, NJ, USA), 2002.

19. N. Max, "Optical models for direct volume rendering," IEEE Transactions on Visualization and Computer Graphics 1(2), pp. 99-108, 1995.

20. J. F. Blinn, "Models of light reflection for computer synthesized pictures," SIGGRAPH Computer Graphics 11(2), pp. 192-198, 1977. 